Bundesgesundheitsbl $2011 \cdot 54: 243-243$

DOI 10.1007/s00103-010-1213-x

Online publiziert: 29. Januar 2011

(c) Springer-Verlag 2011

\title{
4. Mitteilung zur Änderung der Leitlinie zur hygienischen Beurteilung von organischen Beschichtungen im Kontakt mit Trinkwasser (Beschichtungsleitlinie) $^{1}$
}

Das Produkt „Saniline W“ der Firma „Sanivar AG“ wurde für den Einsatzbereich „Rohre mit DN $\geq 80$ mm“ im Kaltwasserbereich mit einer Gültigkeit bis zum 4.02.2014 aufgenommen.

Die Produkte „Epoxidharzgrundierung Friazinc HS (grau)“, „LSE-0o1 NA“, „Hempel's Pipecoating 656 DE“ wurden gestrichen.

Die Gültigkeit des Prüfzeugnisses für das Produkt „Betonkleber GI 166 (grau)“ wurde bis zum 09.03.2015 verlängert.

Die Gültigkeit des Prüfzeugnisses für das Produkt „Dichtungsklebstoff DELO-DUOPOX AD 1997“ wurde bis zum 18.05.2015 verlängert.

Die Gültigkeit des Prüfzeugnisses für das Produkt „Hempel's Pipe-

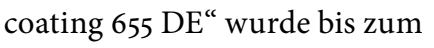
23.11.2014 verlängert.

Die Produkte „Epimid LF-TWB N 300 “ und „Epimid LF-TWB 500“ der Firma Vorrink Stahl- und Betonschutz GmbH \& Co. KG wurden für den Einsatzbereich „Rohre mit DN $\geq 300$ mm" im Kaltwasserbereich mit einer Gültigkeit bis zum 10.03.2014 und 20.08.2014 aufgenommen.
Der Firmenname für die Produkte „Protegol EP 32-97 (beige)“ und „Protegol EP 32-99 (ocker)“ lautet „TIB Chemicals AG“.

Die Gültigkeit des Prüfzeugnisses für das Produkt „Protegol EP 32- 99 (ocker)" wurde bis zum 28.12.2015 verlängert.
Die Gültigkeit des Prüfzeugnisses für das Produkt „Spritzbeschichtung Steulerflake EPM (beige)“ wurde bis zum 15.12.2014 verlängert.

Beschichtungen anderer Bindemittelsysteme

c) für werkseitige Anwendungen (zum Beispiel Pulverlacke):

Das Produkt „Epoxyesterharz banner blue“ wurde gestrichen.

Beschichtungen anderer Bindemittelsysteme

d) für baustellenseitige Anwendungen:

Die Gültigkeit des Prüfzeugnisses für das Produkt „Protegol UR 32-45“ der Firma „TIB Chemicals AG“ wurde bis zum 17.11.2015 verlängert.

1 Umweltbundesamt (2009) Leitlinie zur hygienischen Beurteilung von organischen Beschichtungen im Kontakt mit Trinkwasser (Beschichtungsleitlinie): Bundesgesundheitsbl Gesundheitsforsch Gesundheitsschutz, 52: 960 